\title{
mTOR in FSGS
}

Our data
provide clear
experimental
evidence
for a novel
treatment
regimen
based on
partial mTOR
inhibition

Progression of focal segmental glomerulosclerosis (FSGS) is associated with podocyte loss and hypertrophy of remaining podocytes; however, the mechanisms underlying these processes are unknown. New findings reveal a role for $\mathrm{mTORC1}$ in balancing glomerular maintenance and disease progression in FSGS. "Complete mTOR inhibition or mTOR hyperactivation accelerates glomerulosclerosis progression, whereas moderate mTOR inhibition has a beneficial effect," says Tobias Huber.

Previous studies showing that mTOR regulates podocyte hypertrophy led Huber and colleagues to assess the role of mTOR in glomerulosclerosis. The researchers noted upregulation of mTORC1 target genes in microdissected glomeruli from patients with primary FSGS compared with levels in patients with minimal change disease. Similarly, mTORC1 was activated in podocytes of mice with Adriamycin (ADR)induced nephropathy, and constitutive hyperactivation of mTORC1 led to the development of FSGS-like lesions, highlighting mTORC1 activation as a driver of FSGS. The researchers then assessed the effect of podocyte-specific mTORC1 suppression. Surprisingly, inhibition of mTORC1function by deleting both Raptor alleles in podocytes potentiated the effects of ADR, whereas heterozygous deletion of Raptor ameliorated ADR-induced disease.

To assess the molecular consequences of mTOR signalling in podocytes, Huber and colleagues inhibited mTOR in immortalized human podocytes. High-dose treatment with an mTOR inhibitor led to mitochondrial impairment, whereas low-dose inhibition ameliorated ADR-induced mitochondrial dysfunction. "We found that complete mTOR inhibition shifts cellular energy metabolism towards reduced rates of oxidative phosphorylation and anaerobic glycolysis, whereas prolonged mTOR activation results in metabolic podocyte reprogramming leading to increased cellular stress and dedifferentiation," explains Huber. "Our data provide clear experimental evidence for a novel treatment regimen based on partial mTOR inhibition to prevent progressive glomerulosclerosis and FSGS."

Huber says his future aims are to better understand the downstream effectors of mTOR and their role in podocyte physiology. 